\title{
Direct experimental evidence for very long fission times of super-heavy elements*
}

\author{
M. Morjean ${ }^{1}$, J.L. Charvet ${ }^{2}$, A. Chbihi ${ }^{1}$, M. Chevallier ${ }^{3}$, C. Cohen ${ }^{4}$, \\ D. Dauvergne ${ }^{3}$, R. Dayras ${ }^{2}$, A. Drouart ${ }^{2}$, J.D. Frankland ${ }^{1}$, \\ D. Jacquet ${ }^{5}$, R. Kirsch ${ }^{3}$, M. Laget ${ }^{5}$, P. Lautesse ${ }^{3}$, \\ A. L'Hoir ${ }^{4}$, A. Marchix ${ }^{1}$, L. Nalpas ${ }^{2}$, M. Parlog${ }^{1}$, C. Ray ${ }^{3}$, \\ C. Schmitt ${ }^{3}$, C. Stodel ${ }^{1}$, L. Tassan-Got ${ }^{5}$, \\ C. Volant ${ }^{2}$
}

February 5, 2007

[1] GANIL, DSM/CEA, IN2P3/CNRS ,BP 55027, F-14076 Caen Cedex 5, France

[2] DSM/DAPNIA/SPhN, CEA Saclay, F-91191 Gif/Yvette Cedex, France

[3] IPNL, IN2P3/CNRS, Univ. Lyon 1, 4 rue E. Fermi, F-69622 Villeurbanne Cedex, France

[4] INSP, Univ. Paris 6 et Paris 7,140 rue de Lourmel, F-75015 Paris, France

[5] IPN Orsay, BP 1 F-91406 Orsay Cedex, France

\begin{abstract}
The blocking technique in single crystals has been applied to reaction time measurements for the ${ }^{238} \mathrm{U}+\mathrm{Ge}$ system at $6.1 \mathrm{MeV} /$ nucleon. Backed up with a reaction mechanism analysis using the INDRA $4 \pi$ detector, it provides a direct experimental evidence for $\mathrm{Z}=124$ compound nuclei living longer than $10^{-18} \mathrm{~s}$, indicating thus very high fission barriers for this element.
\end{abstract}

\section{Introduction}

The very large Coulomb energies involved make super-heavy elements (SHEs) extremely unstable against fission $[1,2]$. The liquid drop model predicts vanishing fission barriers for SHEs with atomic number beyond $Z \sim 104$, but shell effects contribute to increase these barriers and very stable nuclei are thus expected in the neighbourhood of closed-shell structures for spherical nuclei. However, the heaviest SHEs can only be formed with sizeable cross-sections by fusion reactions of heavy nuclei leading to compound nuclei at rather high excitation energies and the shell effects progressively vanish with temperature. The instability against fission becomes thus very high and the cross-sections for particle evaporation without fission are experimentally almost unreachable.

Fission is a dynamical process that needs time. Nuclear dissipation is now well known to slow down the fission process [3] and to be responsible for reductions of the statistical fission widths that favor light particle evaporation. At the very beginning of the competition between fission and evaporation, during a transient time, the statistical fission width progressively increases up to a stationary value. During this time, the particle evaporation is strongly favored. Therefore, the excited nuclei evaporate particles (mainly neutrons) and the temperature decreases. The lifetimes of the residual nuclei are thus strongly increased at the end of the transient time. After the transient time, the statistical fission widths are also reduced by the nuclear dissipation [4] and cooling by particle emission is still favored

*Submitted for publication in The European Physical Journal D 
with respect to fission. Typical fission time distributions for highly excited uranium nuclei, calculated within the Bohr and Wheeler approach [5] adapted to take into account viscosity effects with the Kramers formalism[4] and including transient effects, are presented in Fig.1 for various values of the reduced friction parameter $\beta$. Despite the relatively low fission barriers involved (as compared to the fission barriers predicted for doubly magic SHEs at very low excitation energies), the fission time distributions exhibit even for $\beta=2 \times 10^{21} \mathrm{~s}^{-1}$ a sizeable tail at times longer than $10^{-18} \mathrm{~s}$ resulting from the pre-fission emission. The pre-fission emission should play an important role in SHE fission $[6,7,8]$. In reactions between very heavy nuclei, possibly leading to SHE formation, a rather high pre-fission emission has been measured [9] on the average. A progressive shell effect restoration might thus occur, leading to significant increases of the fission barriers and thus of the fission times.

In a recent experiment, very long fission times, longer than $10^{-18} \mathrm{~s}$, have been measured for composite systems formed in the ${ }^{238} U+N i$ reactions at $6.6 \mathrm{MeV} /$ nucleon, a signature of compound nucleus formation with $\mathrm{Z}=120[10,11,12]$. In the present paper, the blocking technique in single crystals has been used to measure reaction times in the ${ }^{238} U+G e$ reactions at $6.1 \mathrm{MeV} /$ nucleon, searching for a direct evidence for $\mathrm{Z}=124$ compound nucleus formation at an excitation energy of about $70 \mathrm{MeV}$.

\section{Blocking technique in single crystals}

The blocking effects in single crystals were discovered in $1965[13,14]$ and since this time have been deeply studied and understood in connection with the closely related channeling effects (for a review see [15]). A very schematic description of this technique is presented in Fig.2. A single crystal is used as a target. When a nuclear reaction occurs between a projectile nucleus and one of the crystal nuclei, a composite system recoiling with the center-of-mass velocity is formed. This system will separate in two fragments at a distance $d$ from the crystal row. A fragment emitted precisely in the direction of this row will be deflected at an angle $\Psi$ with respect to its initial direction by the atomic interactions with the atoms of the crystal. Axial (planar) dips are thus observed in the axis (plane) directions. It can be easily inferred from Fig.2 that the smaller the distance $d$ from the atomic row in which the splitting occurs, the larger the deflection angle $\Psi$ is. Therefore, the filling and the shape of the axial dips are connected to the reaction time. However, a dip evolution is only observed in a limited sensitivity window, between $t_{\min }$ and $t_{\max }$. For very long times, $t>t_{\max }$, the scission may occur at any place in the crystal and no dip is observed. The $t_{\max }$ value depends on the crystal structure, on the lattice constant and on the azimuthal angle of the beam direction with respect to the crystal. When the beam direction is far from any major crystallographic plane, $t_{\max }$ is of the order of the time needed for the recoiling system to travel upon the distance between two adjacent rows. A typical value of $t_{\max }$ is about $5 \times 10^{-17} \mathrm{~s}$ for the system studied in the present paper. The $t_{\min }$ value corresponds to a transverse recoil distance equal to the thermal vibration amplitude of the crystal atoms. It depends on the recoil velocity of the composite system and its direction relative to the crystal axis. If the splitting occurs within the thermal vibration domain, a fragment emitted in the direction of the axis will be strongly deflected by atoms on the row. The dips obtained for times shorter than $t_{\min }$ are thus the deepest and do not present any evolution with the reaction times. This lower time limit has been clearly evidenced for fissions of uranium nuclei excited at up to $600 \mathrm{MeV}$ [16]. A quite sensitive modification of the dips was observed below $250 \mathrm{MeV}$, whereas for higher excitation energies, where the fission times become very short, all the measured dips were quite similar.

The blocking technique is thus a quite direct way, model independent, to get evidence for reaction times longer than $t_{\min }$. This evidence can be immediately reached from raw experimental data: the angular distribution measured with respect to a crystal axis direction (blocking dip) presents at $\Psi=0^{\circ}$ a minimum value $\chi_{\min }$ that decreases with reaction time down to its smallest possible value, reached for times shorter than $t_{m i n}$. Since the $\chi_{\min }$ value is, to first order approximation, only sensitive to time effects for a given crystal (for a demonstration, see for example ref. [15]), an increase of the $\chi_{\min }$ value for a given selection of events is an experimental evidence for reaction times longer than $t_{\text {min }}$ in this selection. For the system studied in the present paper, ${ }^{238} U+G e$ at $6.1 \mathrm{MeV} / \mathrm{nucleon}$, the center-of-mass velocity, the detection angle $\left(\alpha=20^{\circ}\right.$ for the $<100>$ axis considered $)$ and the thermal vibration amplitude in germanium crystals lead to $t_{\min } \sim 1 \times 10^{-18} \mathrm{~s}$. 


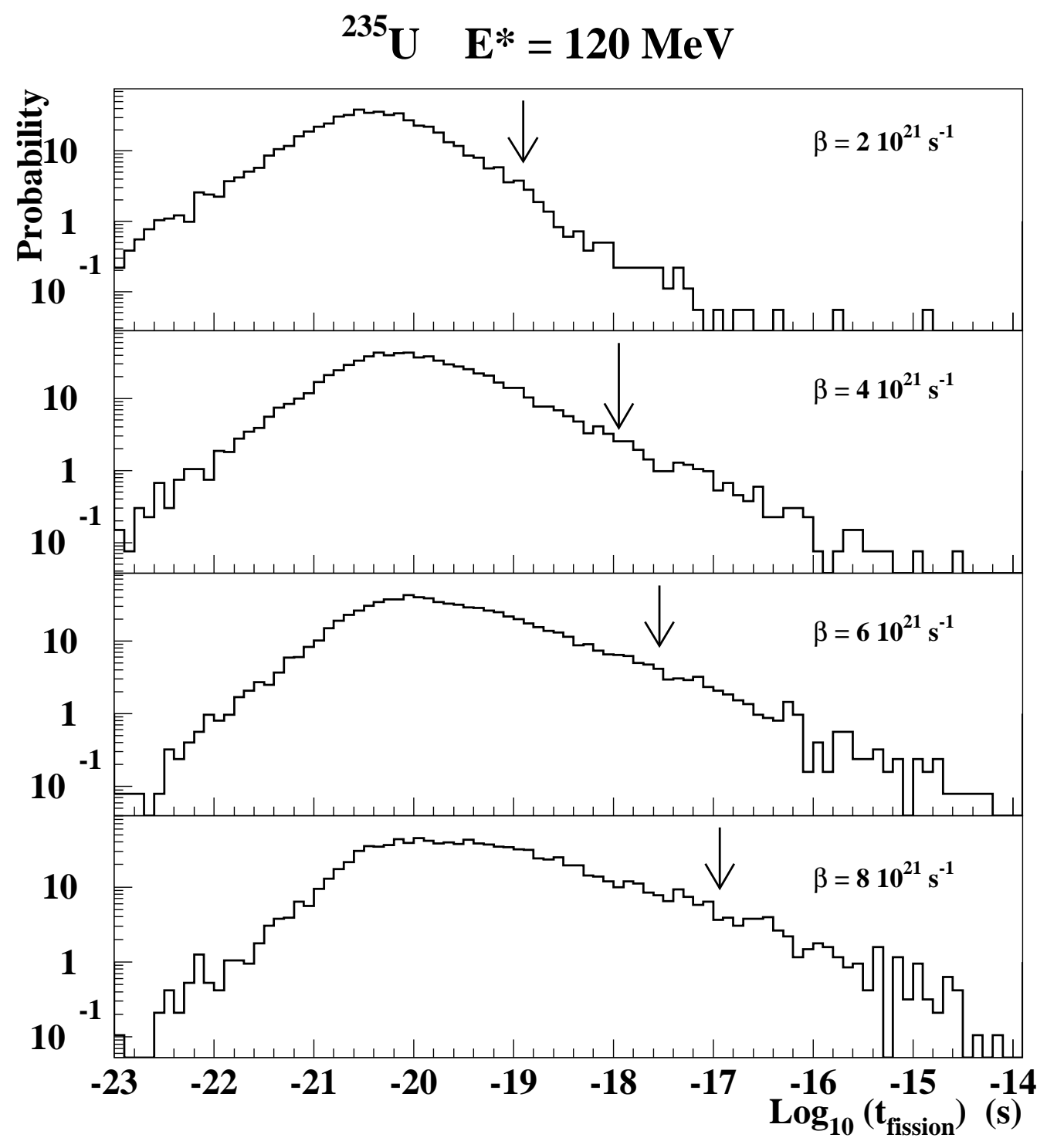

Figure 1: Fission time distributions for uranium nuclei calculated for various reduced friction parameters $\beta$. The probabilities are given in arbitrary units. The arrows indicate the average values of the distributions. 


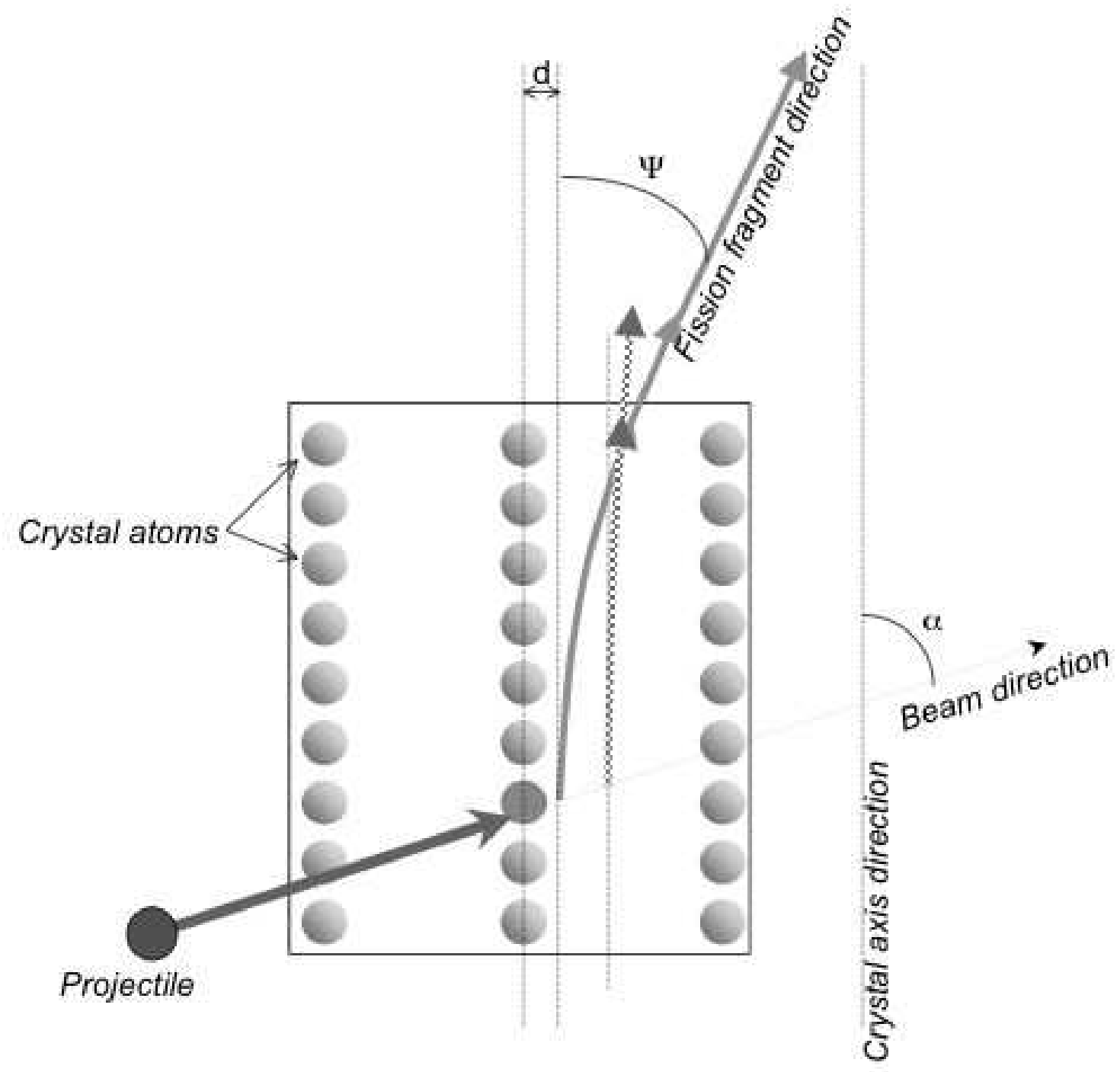

Figure 2: Principle of reaction time measurement by the blocking technique in single crystals. 
For highly fissile nuclei, as shown for example by Fig.1, most of the long lived events that contribute to fill the fast dip (the dip associated to fissions occurring only at times shorter than $t_{\text {min }}$ ) are located in the transition region between $t_{\min }$ and $t_{\max }$ and to a less extent at times longer than $t_{\max }$. For simulated dips associated with given fission times, a continuous evolution is of course observed between the fast dip at $t_{\min }$ and a flat distribution at $t_{\max }$. Thus, the measured dip depends strongly on the actual fission time distribution since, despite their weaker effect on the dip characteristics, the shorter time events with $t>t_{\min }$ are much more probable than the longer time ones. However, the distribution is a priori unknown, highly model and parameter dependent (see for instance Fig.1). The measured dip thus contains information on the time distribution above $t_{\text {min }}$ that might be inferred from an unfolding procedure in an ideal experiment involving very high statistics. However, in real experiments, due to limited statistics and experimental uncertainties, measured dips can be reproduced (see [16]) in a satisfactory way by very different time distributions, implying quite different percentages of long time events. Therefore, in these experiments, although the presence of fission components at times longer than $t_{\min }$ are clearly signalled by an increase of $\chi_{\min }$, the percentage of events corresponding to times longer than $t_{\min }$ (or longer than $t_{\max }$ ) cannot be extracted in a unique way.

The blocking technique provides however a valuable test for the fission time distributions and, especially, their average values. In a first approach, the distributions that reproduce through simulations the measured dip $[16,20,21]$ can be characterized by their average values and their variances. Since the decay of the nuclei must follow the radioactive decay law, a fit performed assuming an exponential shape for the fission time distribution provides us with the smallest variance and the shortest average time possible. The other extreme limits are found assuming a two-component distribution with fission events at times shorter than $t_{\min }$ and fission events at a time $t_{\text {lim }}$ longer than $t_{\max }$. In the case of highly fissile nuclei, a maximum value for $t_{\text {lim }}$ can be obtained from the time at which the $\gamma$-ray emission widths almost fill the total statistical decay width $[22,23]$, the $\gamma$-rays removing thus all the residual excitation energy (this assumption of a two-component distribution is quite unrealistic since a competition between fission and $\gamma$-ray emission would lead to high residue production rate which is not observed experimentally; this rough assumption is only made to get an upper limit). For such a two-component distribution, the average time is governed by the events at $t_{\text {lim }}$; the fission time assumed for the events at times shorter than $t_{\text {min }}$ does not affect significantly the average value. The two extreme distributions (exponential and two-components) thus provide us with the dispersion of the possible time distribution average values. The average fission times of uranium nuclei have been determined following this procedure with an overall uncertainty of a factor 3 [16] obtained for the realistic value of $t_{\text {lim }}$ considered, leading to time values in good overall agreement with those inferred from a quite different atomic clock based on X-ray detection [17, 18]. Despite the rather large uncertainties resulting from this procedure, the blocking technique gives decisive insights since the average calculated fission times vary, depending on the model and on the parameters considered, by several orders of magnitude (see for example Fig.1 ). Such an analysis is in progress for the present experiment. However, the existence of composite systems surviving at times longer than $10^{-18} \mathrm{~s}$ can be directly inferred from a $\chi_{\min }$ analysis. In the following, we shall thus focus on this analysis. In addition, we shall show from an analysis of the associated reaction mechanisms that very long reaction times arise from compound nucleus fissions.

\section{Evidence for $\mathrm{Z}=124$ compound nucleus formation}

In reactions between two heavy nuclei, the formation of SHE compound nuclei followed by fission is difficult to sign due to the presence of non-equilibrium fast processes (quasi-fission) that have exit-channel characteristics very similar to the ones associated with the fusion-fission process[19]. In contrast to other approaches that apply more or less arbitrary selections in order to discriminate between fusion-fission and quasi-fission, the blocking technique in single crystals provides us with an unambiguous signature of fusion-fission reactions: reaction times longer than about $10^{-18} \mathrm{~s}$, at least two or three orders of magnitude longer than the quasi-fission reactions [19], can be immediately identified in the raw data due to the lower sensitivity limit $t_{\text {min }}$ described in the previous chapter.

The experimental set-up has been already described in references $[11,12]$. A $2 \mu \mathrm{m}$ thick germa- 
nium single crystal was bombarded by $6.1 \mathrm{MeV} /$ nucleon ${ }^{238} \mathrm{U}$ ions accelerated by the GANIL facility. Specially designed telescopes aiming at measuring the blocking effects provided us with the atomic numbers $\mathrm{Z}$ and the energy of nuclei detected in an angular range covering $\pm 1.2^{\circ}$ around $20^{\circ}$. An angular resolution of the order of $2 \times 10^{-2}$ degrees, an energy resolution better than $5 \%$ and an atomic number resolution of the order of 2 units for the heaviest nuclei was achieved with these telescopes. In addition, the charged products in coincidence with the blocking telescopes were detected and identified by INDRA [24], a $4 \pi$ charged product detection array, in order to have a good control on the reaction mechanisms involved and to prevent any bias on the results arising from incomplete fusion reactions, from sequential fission of excited projectile-like fragments...

A $\Delta E-E$ identification matrix measured at $20^{\circ}$ by one of the blocking telescopes is presented in Fig.3. Four zones, labeled from a to d, are defined by contours in this figure. The blocking patterns corresponding to each of these zones are presented in Fig.4. The full curves drawn in Fig.4 are Gaussian fits to the dips. It has been carefully checked that the function used for the fits affects only weakly the relative evolution of the $\chi_{\min }$ values. The zone defined at the lowest $\Delta E$ values (label d) is populated by target-like fragments arising from quasi-elastic and deep-inelastic scattering. The $\chi_{\text {min }}$ value of this dip is the lowest possible value in the present experiment since the associated mechanisms obviously correspond to reaction times much shorter than $t_{\min }$ (for an ideal germanium single crystal, a $\chi_{\min } \approx 0.025$ should be actually obtained; the higher $\chi_{\min }$ value measured here indicates only that the crystal used is not perfect, as confirmed by specific measurements done for pure elastic scattering of the projectile). A similarly low $\chi_{\text {min }}$ value (within the statistical uncertainties) is obtained, as expected, in Fig4-a for the fragments located inside the zone a, corresponding to highly excited projectile-like fragments arising from deep-inelastic reactions and which survived fission. The $\chi_{\min }$ values obtained for zones a and $\mathrm{d}$ are the same as the one obtained by selecting only target quasi-elastic scattering. Fragments arising from deep-inelastic collisions are excited much above the particle emission thresholds, and thus emit light particles (mainly neutrons). Therefore, the small and similar $\chi_{\min }$ values measured for deep-inelastic and quasi-elastic scatterings show that light particle emission from a fragment does not affect significantly the $\chi_{\min }$ of the dips, as confirmed by simulations. The excited projectile-like fragments most likely undergo fission. The zone c in Fig.3 is dominantly populated by the fragments arising from this sequential fission of uranium-like nuclei. A much higher $\chi_{\min }$ value $(\sim 0.4)$ is found as expected [16] for these slow fissions (Fig.4-c). The last zone (b) in Fig.3 corresponds to asymmetric binary splitting of the composite system. This zone is commonly assumed to be dominantly populated by quasi-fission events. However, Fig.4-b shows for this zone a $\chi_{\min } \approx 0.2$, indicating a significant proportion of fission fragments arising from nuclei living longer than $10^{-18} \mathrm{~s}$, either compound nuclei with $\mathrm{Z}=124$, nuclei formed in incomplete fusion reactions or projectile-like fragments undergoing sequential fission. The striking difference in shape of the 4 dips presented in Fig. 4 arises from the very different atomic numbers (from about $\mathrm{Z}=90$ for zone a down to about 20 for zone d) and from the quite different energy ranges involved: indeed, the half-angular width of a blocking dip is roughly proportional to $(Z / E)^{1 / 2}[13]$.

The reaction mechanism(s) associated to the long living nuclei highlighted in zone $\mathrm{b}$ have been studied with the help of the coincident charged products measured by INDRA. The folding angle of the coincident fission fragment detected by INDRA is in good agreement with what is deduced from the Viola systematics [25] for compound nucleus fission. From this folding angle, a sequential fission process of the projectile-like fragment can be excluded. Furthermore, a very low multiplicity $\left(\ll 10^{-2}\right)$ of coincident intermediate-mass fragments $(2<Z<32)$ is measured by INDRA. The folding angle is however a weakly sensitive probe for incomplete fusion reactions in which light particles escape from the composite system. A multiplicity $M_{l c p}<0.5$ of coincident light charged particles $(Z<3)$ (a more precise determination is under progress) is indeed measured, but removing from zone $b$ all the events associated with light charged particles does not modify significantly the observed $\chi_{\min }$ value. Therefore, incomplete fusion reactions can also be ruled out and the composite systems that live more than $10^{-18}$ s can only be compound nuclei with $Z=124$, a first evidence for very high fission barriers for this nucleus.

The $\chi_{\min }$ value associated to zone $\mathrm{b}$ increases by roughly 0.1 as compared to the one associated to very fast processes; this increase provides directly the lower limit of the fraction of fusion-fission events. The longer the reaction time, the weaker the blocking effect experienced by the corresponding 


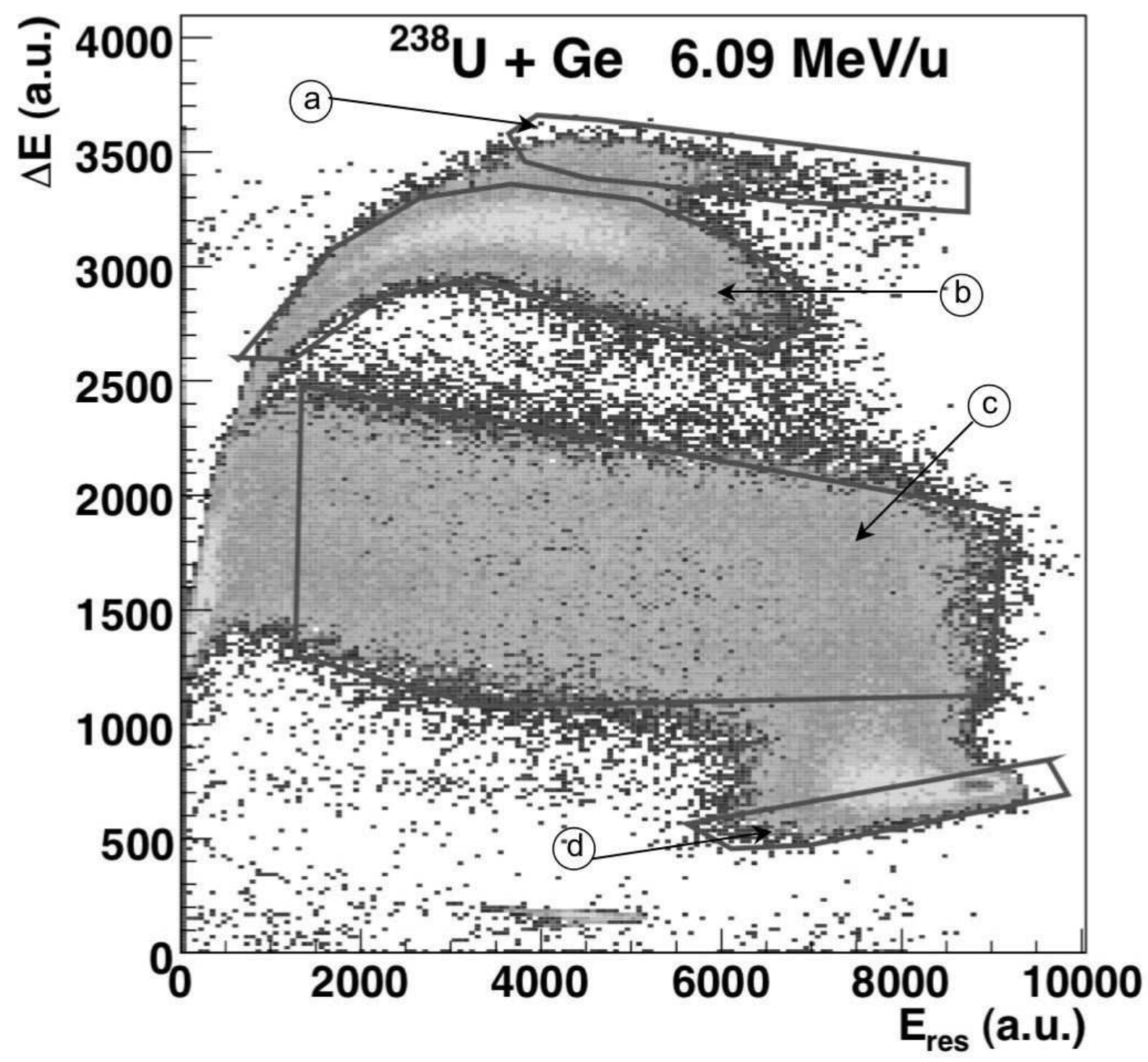

Figure 3: Energy loss $\Delta E$ versus residual energy $E_{r e s}$ identification map measured at 20 degrees. Four zones corresponding to different dominant reactions mechanisms are delimited by contours. 


\section{$238 \mathrm{U}+\mathrm{Ge} \quad 6.09 \mathrm{MeV} / \mathrm{u}$}

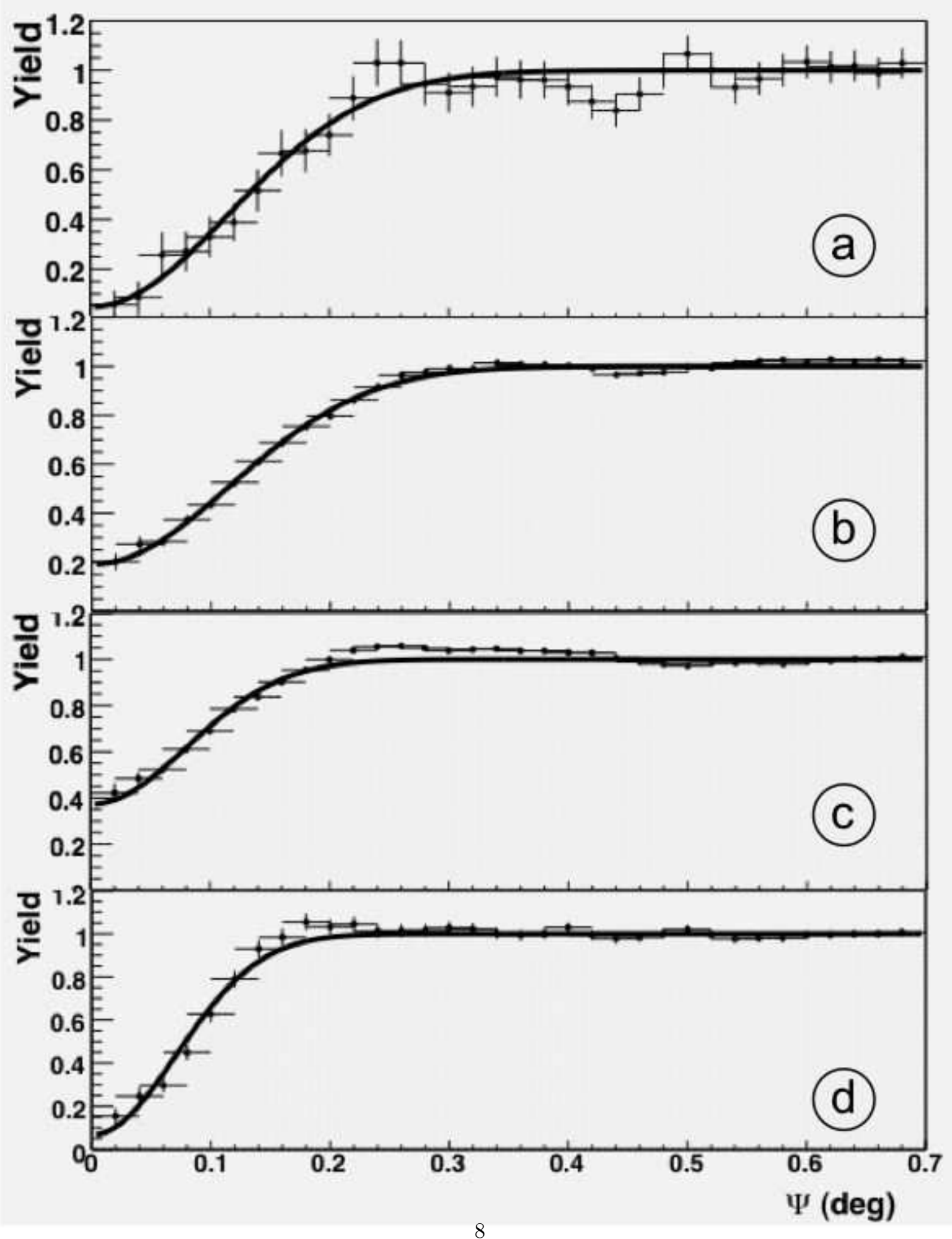

Figure 4: Blocking dips integrated around the $<100>$ axis of the Ge crystal for the 4 zones defined in Fig.3. The full curves correspond to Gaussian fits of the dips. 
fragments, and the stronger their contribution to the overall increase of $\chi_{\text {min }}$. Thus, assuming that all the fission fragments responsible for the observed $\chi_{\min }$ increase experience no blocking effect at all (very long reaction times $t>t_{\max }$ ) leads to a percentage of fusion-fission events of about $10 \%$. However, a non-negligible proportion of the fission events following complete fusion is at times between $t_{\min }$ and $t_{\max }$. The corresponding fission fragments experience thus some blocking and contribute in a weaker way to the overall increase of $\chi_{\min }$ : the actual percentage of fusion-fission events is higher than $10 \%$. This surprisingly high weight of fusion followed by asymmetric fission might arise from the large detection angle in the center-of-mass frame: the cross-section for the quasi-fission process seems to have strongly decreased at this angle. Time measurements at more forward angles would bring valuable pieces of information, but these measurements seem to be out of range, due to the high counting rate of elastic scattering inside the grazing angle.

\section{Conclusion}

The long fission times observed in raw experimental results obtained using the blocking technique in a single crystal, combined with a reaction mechanism analysis, provide us with quite direct evidence for the formation of compound nuclei with $\mathrm{Z}=124$ in the ${ }^{238} \mathrm{U}+\mathrm{Ge}$ at $6.1 \mathrm{MeV} /$ nucleon reactions. Considering its rather high excitation energy $\left(E^{*} \sim 70 \mathrm{MeV}\right)$, the $\mathrm{Z}=124$ nucleus formed must have a high fission barrier enhanced by large shell effects that might be progressively restored by fast prefission emission. This result is a clue to an island of stability in the vicinity of this element. New fission time measurements for neighboring elements are needed to get a better delimitation of this stability island.

\section{References}

[1] S. Hofmann and G. Münzenberg, Rev. Mod. Phys. 72, (2000) 733

[2] Yu.Ts. Oganessian et al., Nature 400 (1999) 242

[3] D. Hilscher and H. Rossner, Ann. Phys. (Paris) 17, (1992) 471

[4] H.A. Kramers, Physica (Utrecht) 7, (1940) 84

[5] N. Bohr and J.A. Wheeler., Phys. Rev. 56, (1939) 426

[6] Y. Abe et al., J. Phys. G 23, (1997) 1275

[7] Y. Aritomo et al., Phys. Rev. C 59, (1999) 796

[8] M. Ohta et al., Proc. of Tours Symposium on Nuclear Physics II, (World Scientific ,1994) 480

[9] M.G. Itkis et al, Nucl. Phys. A 734, (2004) 136

[10] D. Jacquet et al., in preparation

[11] D. Jacquet et al., Proceedings of the Int. Conf. on Reaction Mechanisms and Nuclear Structure at the Coulomb Barrier, March 19-23 2006, Venezia, Italy AIP 853 (2006) 239

[12] A. Drouart et al., Proceedings of the Int. Symposium on Exotic Nuclei, Peterhof, July 5-12 2004 (World Scientific, Singapore 2005) 192

[13] D.S. Gemmell and R.E. Holland, Phys. Rev. Lett. 14, (1965) 945

[14] A.F. Tulinov,Sov. Phys. Doklady 10, (1965) 463

[15] D.S. Gemmell, Rev. Mod. Phys. 46, (1974) 129

[16] F. Goldenbaum et al., Phys. Rev. Lett. 82, (1999) 5012

[17] J.D. Molitoris et al., Phys. Rev. Lett. 70, (1993) 537

[18] H.W. Wilschut and V.L. Kravchuk, Nucl. Phys. A 734, (2004) 156

[19] J. Töke et al., Nucl. Phys. A, 440, (1985) 327 
[20] F. Barrué et al., NIM B, 193, (2002) 852

[21] C. Cohen and D. Dauvergne, NIM B, 225, (2004) 40

[22] F. Puhlhofer, Nucl. Phys. A, 280, (1977) 267

[23] K.A. Snover, Ann. Rev. Nucl. Part. Sci., 36, (1986) 545

[24] J. Pouthas et al., NIM A, 357, (1995) 41

[25] V.E. Viola et al., Phys. Rev. C 31, (1985) 1550 